'Universidade da Integração Internacional da Lusofonia AfroBrasileira (UNILAB), Av. da Abolição, 3 Centro, CEP 62790-000, Redenção, $\mathrm{CE}$, Brasil

*autor correspondente $\nabla$ sousamuriloalmeida@gmail.com

\title{
Sustentabilidade de agroecossistemas: estudo de caso em propriedades rurais no maciço de Baturité/CE
}

\author{
Sustainability of agroecosystems: case study on rural \\ properties in the massif of Baturité / CE
}

Murilo Sousa Almeida ${ }^{*}$, Maria Brenna Mendes Cunha' ${ }^{1}$ Jose Abel Aguiar da Silva Paz ${ }^{1}$ Filipe Eugênio Rodrigues Silvestre ${ }^{1}$

RESUMO: A sustentabilidade na agricultura pode ser mensurada através de indicadores, elaborados para este fim. O presente trabalho avaliou a sustentabilidade em dois agroecossistemas localizados no Maciço de Baturité, estado de Ceará, pela aplicação da metodologia MESMIS, com o uso de indicadores econômicos, socioculturais e ambientais. O sistema 2 baseado em produção agrícola e pecuária alcançou os melhores níveis de sustentabilidade, ao passo que o agroecossistema 2 especializado na produção agrícola obteve os menores índices de sustentabilidade. A sustentabilidade econômica tem maior déficit nos sistemas estudados nas cidades de Baturité/ CE e Barreira/CE. A diversificação de cultivos aliado ao uso mais conservacionista dos recursos disponíveis, com a expansão mercadológica de ambos os sistemas podem auxiliar para a mudança dos níveis de sustentabilidade dos agroecossistemas

PALAVRAS-CHAVE: Agricultura familiar, agroecologia, indicadores.
ABSTRACT: Sustainability in agriculture can be measured through indicators, designed for this purpose. The present work evaluated sustainability in two agroecosystems located in the Massif de Baturité, state of Ceará, by applying the MESMIS methodology, using economic, socio-cultural and environmental indicators. The system 2 based on agricultural and livestock production achieved the best levels of sustainability, while the agroecosystem 2 specialized in agricultural production obtained the lowest levels of sustainability. Economic sustainability has a greater deficit in the systems studied in the cities of Baturité / CE and Barreira $/ C E$. The diversification of crops combined with the more conservative use of available resources, with the market expansion of both systems can help to change the levels of sustainability of agroecosystems

KEYWORDS: Family farming, agroecology, indicators.

\section{Introdução}

$\mathrm{O}$ crescimento em progressão das atividades agrícolas na ocupação da população rural e a complexidade dos fatores que influenciam nesse comportamento são constatações de novas funções do meio rural, as mudanças nas famílias rurais e nas explorações agropecuárias; as oscilações do mercado de trabalho urbano e rural, além de introdução de novas tecnologias sociais e ambientais no sistema de produção.

Essas mudanças que incluem a utilização de novas sementes hibridas, uso de insumos industriais, mecanização e práticas que permitiram uma progressão positiva da atividade agrícola. Logo ocorre uma diversidade biológica, a redução dos recursos florestais, o comprometimento da qualidade dos recursos hídricos e do solo estão entre os mais aparentes. Gallo et al. (2016).

Nesse contexto é relevante destacar o papel que a agricultura familiar tem na produção de alimentos consumidos pela sociedade Portanto, seu fortalecimento não só pelo respeito que devem merecer como grupo social, mas também pelo 
apoio à segurança alimentar, à produção de matérias-primas, ao desenvolvimento local e regional e à conservação da natureza Borges et al. (2020)

As adversidades da gestão em propriedades familiares estão relacionadas estão ligadas a alguns aspectos fundamentais: inadequação das ferramentas gerenciais existentes à realidade da agricultura familiar; descapitalização dos pequenos agricultores que não podem ter acesso e beneficiar-se das modernas tecnologias de informação; baixo nível de educação formal dos agricultores familiares; falta de uma cultura que crie um ambiente propicio à adoção de novas tecnologias de gestão e, finalmente, o não conhecimento em relação aos níveis de sustentabilidade das suas respectivas propriedades Silva et al. (2016)

Porém, ainda é escasso o conhecimento em relação aos níveis de sustentabilidade das atividades exercidas em unidades produtivas familiares Gallo et al. (2014). A metodologia de pesquisa em sistemas de produção constitui-se como um instrumento capaz de auxiliar a construção do desenvolvimento rural sustentável, já que, segundo Gallo et al. (2014), ela permite entender o ambiente ecológico e socioeconômico no qual as atividades ocorrem, incluindo pequenos produtores como colaboradores e beneficiários e estimulando a promoção de tecnologias apropriadas.

Dessa forma, os indicadores não só direcionam para implantação de agroecossistemas mais adaptados, através da análise dos dados, como também fornecem informações que viabilizam ferramentas políticas e estratégias de planejamento para elevar o nível de sustentabilidade das propriedades. Além disso, apresentam informações relevantes para produtores da área estudada que apresentam condições semelhantes em suas unidades de produção. Nessa perspectiva, os indicadores de sustentabilidade na agricultura familiar são variáveis que apresentam uma descrição quali-quantitativa do agroecossistema, contemplado três dimensões: social, econômica e ambiental Gonçalves et al. (2016).

Diante do exposto, o presente artigo tem como objetivo avaliar os parâmetros de sustentabilidade de dois agroecossistemas de base familiar na macrorregião do Maciço de Baturité/CE, visando estabelecer um comparativo entre seus sistemas de manejo através de indicadores sociais, econômicos e ambientais.

\section{Material e Métodos}

A área de estudo do Maciço de Baturité, que por sua vez foi dividido em três micro territórios, são: micro território I composto pelos municípios de Palmácia, Pacoti, Guaramiranga, Mulungu e Aratuba, pelo micro território II formado pelos municípios de Baturité, Capistrano, Itapiúna, Aracoiaba, Redenção e Acarape. E pelo Micro Território III que contempla os municípios de Ocara e Barreira. O território Maciço de Baturité possui uma área de $3.709 \mathrm{~km}^{2}$ (INSTITUTO DE PESQUISA E ESTRATÉGIA ECONÔMICA DO CEARÁ, 2006). Quanto à distribuição da população entre as áreas rural e urbana, a maior incidência está na área rural com 54,84\%; enquanto na urbana concentra-se $45,16 \%$ da população total (INSTITUTO BRASILEIRO DE GEOGRAFIA E ESTATÍSTICA, 2020).

A pesquisa foi realizada no segundo semestre de 2019, nos municípios de Barreia e Baturité. Os dados foram coletados em dois agroecossistemas seguindo a metodologia MESMIS. Um agroecossistema é definido como um sistema agrícola, descrito em termos de estrutura e função. Assim, a avaliação necessariamente parte da descrição dos limites dos sistemas, dos seus subsistemas e elementos constituintes, e das inter-relações entre estes elementos, bem como das entradas, saídas e perdas de energia e materiais.

A estrutura básica do MESMIS define que a sustentabilidade pode se expressar em três dimensões: ecológica, econômica e sociocultural. Estas três dimensões se relacionam com cinco atributos da sustentabilidade em agroecossistemas Masera et al. (2000). São eles:

- Produtividade: representada por diversos indicadores como ingressos monetários, rendimento do trabalho e/ou da terra, produção de biomassa, entre outros.

- Estabilidade: é a propriedade do agroecossistema em manter em equilíbrio estável ao longo do tempo, isto é, manter suas funções de produção de biomassa vegetal e ciclagem de nutrientes para fins de fertilidade do solo, e a população de microrganismos, entre outros.

- Adaptabilidade: expressa se o sistema está se ajustado aos diversos fatores externos, e se pode gerar benefícios e/ou prejuízos mesmo com mudanças de longo prazo.

- Equidade: analisar a repartição das externalidades positivas e negativas entre os diversos agentes envolvidos no agroecossistema, e também com a sociedade como um todo.

- Autonomia: avaliar em que medida determinado agroecossistema é capaz de definir seus objetivos, prioridades e estratégias e se consegue de forma autônoma alcançar estes objetivos.

Os agroecossistemas de referência estudados foram: (a) um agroecossistema misto, baseado em cultivos anuais e em hortaliças diversificadas (b) um sistema misto com cultivos anuais, hortaliças diversificadas, além de sistema de produção leiteira de médio porte. De acordo com o MESMIS, foram desenvolvidos indicadores para a avaliação (Tabela 1), abordando os atributos e as três dimensões básicas da sustentabilidade (ambiental, social e econômica). Os descritores e indicadores elaborados foram:

$\mathrm{Na}$ avaliação, foram abordadas as seguintes etapas: a) seleção de indicadores de sustentabilidade; b) estudo detalhado das atividades exercidas na unidade produtiva, através da identificação dos sistemas de manejo e suas características no contexto econômico, social e ambiental; c) mensuração dos indicadores de sustentabilidade; d) levantamento dos pontos críticos dos agroecossistemas; e) análise descritiva dos resultados; f) propostas de ações para a afim de fortalecer a sustentabilidade dos sistemas de manejo Guimarães et al. (2015).

Para que a discussão da sustentabilidade aporte elementos práticos mais além da retórica, é necessário desenvolver marcos conceituais e ferramentas de fácil aplicação para avaliação da sustentabilidade, e também para o planejamento de agroecossistemas de elevada complexidade.

A utilização da metodologia busca, por intermédio de uma abordagem comparativa entre distintos sistemas de produção e/ou recortes temporais, avaliar os níveis de sustentabilidade 
Tabela 1. Descritores e indicadores de sustentabilidade aplicados na propriedade.

\begin{tabular}{|c|c|c|c|}
\hline Atributos & Descritores & Indicadores & Dimensão \\
\hline \multirow{3}{*}{ Produtividade } & Rendimentos & Ingressos monetários & Econômica \\
\hline & \multirow{2}{*}{ Eficiência } & Eficiência de utilização do solo & Ecológica \\
\hline & & Eficiência de utilização do trabalho familiar & Sociocultural \\
\hline \multirow{6}{*}{ Resiliência/Estabilidade } & \multirow{3}{*}{ Diversidade } & Diversidade de cultivos Ecológica & Ecológica \\
\hline & & Presença do subsistema de criação animal & Ecológica \\
\hline & & Diversidade de canais de comercialização Econômica & Econômica \\
\hline & \multirow{3}{*}{ Fragilidade do sistema } & Erosão do solo Ecológico & Ecológica \\
\hline & & Controle sobre o preço dos produtos & Econômica \\
\hline & & Presença de jovens & Sociocultural \\
\hline \multirow{2}{*}{ Adaptabilidade } & \multirow{2}{*}{ Capacidade de mudança } & Existência de pluriatividade & Econômica \\
\hline & & Intercambio de conhecimentos & Sociocultural \\
\hline Equidade & Distribuição de benefícios & Estabelecimento de relações cidade-campo & Sociocultural \\
\hline \multirow{3}{*}{ Autonomia } & \multirow{3}{*}{ Nível de independência } & Uso de insumos externos & Ecológica \\
\hline & & Produção para autoconsumo & Econômica \\
\hline & & Uso de conhecimentos locais & Sociocultural \\
\hline
\end{tabular}

Peano et al. (2015). Mais do que simplesmente apontar o grau de sustentabilidade destes agroecossistemas, a aplicação dos indicadores permite reconhecer os pontos críticos e falhos na estrutura e funcionamento dos mesmos, fomentando propostas de intervenção para o incremento dos níveis de sustentabilidade Alves et al. (2016)

Ao total foram criados 15 indicadores, sendo 5 econômicos, 5 ecológicos e 5 da dimensão sociocultural, distribuídos em 7 descritores associados aos atributos da sustentabilidade dos agroecossistemas. Já que os indicadores são de grandezas e unidades distintas, foi necessário efetuar uma normalização destes, isto é, integrá-los em uma matriz comum de análise. Assim, cada indicador recebeu um valor de um a três, permitindo, posteriormente, comparações entre os atributos e as dimensões da sustentabilidade.

Os parâmetros (tabela 2) foram pensados tendo em conta um agroecossistema hipotético, com um nível elevado de sustentabilidade. Os dois agroecossistemas de estudo foram avaliados em comparação com este "sistema modelo". A nota mínima permitida pelos indicadores elaborados é 15 , e a máxima possível é 45 .

Depois de atribuídas as notas aos indicadores para cada um dos dois agroecossistemas, se efetuou uma análise de síntese, com a criação de Índices de Sustentabilidade para as três dimensões (ecológica, econômica e sociocultural), somando os valores dos indicadores em cada dimensão e dividindo-os por cinco. Os gráficos foram representados no formato radial visando favorecer a interpretação dos dados de cada um dos indicadores utilizados na avaliação.

\section{Resultados e Discussão}

\section{Agroecossistema 1 - Baturité/CE}

Este agroecossistema (Figura 1) é composto por uma família (cinco pessoas) que detém a propriedade a mais de quinze anos. São provenientes de Baturité, onde trabalham como autônomos no comércio e uma pessoa possui experiência prévia com agricultura. A propriedade de 10 hectares é ocupada com diversos cultivos: banana, laranja, limão, macaxeira, coentro, cebolinha. O componente animal no agroecossistema é uma produção secundária sem fins lucrativos e a produção possui canais de comercialização e autoconsumo. A análise da sustentabilidade foi a seguinte:

$\mathrm{O}$ agroecossistema apresentou uma nota de 25 para os indicadores, em uma média de 1,6 (25/15). Assim, é um sistema de baixa sustentabilidade. Os únicos indicadores com notas máximas foram erosão do solo e diversidade de cultivos. Ambos interligados com uma grande rotação de culturas de diferentes famílias botânicas auxiliando na saúde do solo, principalmente contra a erosão do solo.

Como pontos críticos do agroecossitemas podemos citar a falta de controle de preços da produção a ser comercializada, problema esse citado por Medeiros et al. (2012) que a maioria dos pequenos produtores rurais não realizam nenhum tipo de controle de produção, prejudicando seus índices financeiros. Esse indicador liga-se também a falta de relações campo-cidade, tendo como base apenas Centrais Estudais de Abastecimento (CEASA) como centro referência de operações comerciais.

Outro fator importante é a evasão de jovens na propriedade, o que afeta a mão obra. Essa perda está ligada à possível falta de interesse nas práticas agrícolas ligadas à família, debatida por Panno e Dessimon Machado (2014). Esse desinteresse pode estar ligado à falta de políticas públicas para o jovem no campo, a não identificação com atividades agrícolas ou ao pouco espaço experiência para o jovem.

A propriedade contém alta entrada de insumos externos, sejam nas formas de adubos químicos, sementes para plantio ou agrotóxicos. A grande demanda desses materiais faz com que a propriedade seja altamente dependente, fato que que contrapõe a ideia de sustentabilidade. A produção pecuária de pequeno porte sofre com as más instalações zootécnicas, que prejudicam a produção final de leite. 
Tabela 2. Mensuração, parâmetros e notas para os indicadores escolhidos.

\begin{tabular}{|c|c|c|c|}
\hline \multirow{3}{*}{ Ingresso monetário } & \multirow{3}{*}{$\begin{array}{l}\text { Ingressos (em R\$) } \\
\text { mensais/família }\end{array}$} & \multirow{3}{*}{$\begin{array}{l}\text { Salário mínimo R\$ 465,00 } \\
\text { (ano base 2009) }\end{array}$} & 01: < 1 S.M. \\
\hline & & & 02: 1 S.M. até 3 S.M. \\
\hline & & & 03: > 3 S.M. \\
\hline \multirow{3}{*}{ Eficiência de utilização do solo } & \multirow{3}{*}{$\begin{array}{l}\text { \% solo coberto por cultivos } \\
\text { (temporários e permanentes) }\end{array}$} & \multirow{3}{*}{$\begin{array}{c}\text { Pelo menos } 50 \% \text { do solo } \\
\text { utilizado. }\end{array}$} & $01:<50 \%$ \\
\hline & & & 02: $50 \%$ até $90 \%$ \\
\hline & & & $03:>90 \%$ \\
\hline \multirow{3}{*}{$\begin{array}{l}\text { Eficiência de utilização do } \\
\text { trabalho familiar }\end{array}$} & \multirow{3}{*}{$\begin{array}{c}\mathrm{N}^{0} \text { pessoas que trabalham no } \\
\text { lote/ } \mathrm{N}^{0} \text { pessoas da família aptas } \\
\text { ao trabalho }\end{array}$} & \multirow{3}{*}{ Pelo menos 50\% } & $01:<50 \%$ \\
\hline & & & 02: $50 \%$ até $80 \%$ \\
\hline & & & $03:>80 \%$ \\
\hline \multirow{3}{*}{ Diversidade de cultivos } & \multirow{3}{*}{$\mathrm{N}^{0}$ cultivos principais } & \multirow{3}{*}{$\begin{array}{l}\text { Mais que duas espécies } \\
\text { principais cultivadas }\end{array}$} & $01: \leq 2$ cultivos \\
\hline & & & 02: 2 até 5 cultivos \\
\hline & & & $03:>5$ cultivos \\
\hline \multirow{3}{*}{$\begin{array}{l}\text { Presença do subsistema de } \\
\text { criação animal }\end{array}$} & \multirow{3}{*}{$\begin{array}{l}\text { Presença do subsistema de } \\
\text { criação animal }\end{array}$} & \multirow{3}{*}{ Sim } & 01: Não há animais \\
\hline & & & 02: Há uma exploração animal \\
\hline & & & 03: > 1 exploração \\
\hline \multirow{3}{*}{$\begin{array}{l}\text { Diversidade de canais de } \\
\text { comercialização }\end{array}$} & \multirow{3}{*}{$\mathbf{N}^{0}$ de canais de comercialização } & \multirow{3}{*}{ Mais que dois canais principais } & $01: \leq 2$ canais \\
\hline & & & 02: 2 até 4 canais \\
\hline & & & 03: $>4$ canais \\
\hline \multirow{3}{*}{ Erosão do solo } & \multirow{3}{*}{ Observação da erosão no campo } & \multirow{3}{*}{ Sem sinais de erosão } & 01: muita erosão \\
\hline & & & 02: pouca erosão \\
\hline & & & 03: sem erosão \\
\hline \multirow{3}{*}{$\begin{array}{c}\text { Controle sobre o preço dos } \\
\text { produtos }\end{array}$} & \multirow{3}{*}{ Intensidade de controle } & \multirow{3}{*}{ Capacidade de controlar o preço } & 01: sem controle \\
\hline & & & 02: pouco controle \\
\hline & & & 03: muito controle \\
\hline \multirow{3}{*}{ Presença de jovens } & & & 01: sem jovens \\
\hline & $\begin{array}{l}\text { Desejo de permanência dos } \\
\text { jovens na agricultura }\end{array}$ & Jovens trabalhando no lote & $\begin{array}{c}\text { 02: jovens no lote, mas sem } \\
\text { trabalho }\end{array}$ \\
\hline & & & 03: jovens trabalhando no lote \\
\hline & & & $\begin{array}{l}\text { 01: Trabalho não-agrícola fora } \\
\text { do lote }\end{array}$ \\
\hline Existência de pluriatividade & $\begin{array}{c}\text { Existência de trabalhos não- } \\
\text { agrícolas }\end{array}$ & Pluriatividade dentro do lote. & 02: sem pluriatividade \\
\hline & & & $\begin{array}{l}\text { 03: trabalho não-agrícola no } \\
\text { lote. }\end{array}$ \\
\hline & & & 01: sem intercâmbio \\
\hline Intercâmbio de conhecimentos & Intercâmbio entre agricultores & Intercâmbio frequente & 02: pouco intercâmbio \\
\hline & & & 03: intercâmbio frequente \\
\hline Estabelecimento de relações & Proximidade nas relações & & $\begin{array}{l}\text { 01: sem intercâmbio (apenas } \\
\text { relações comerciais) }\end{array}$ \\
\hline campo-cidade & campo-cidade & Intercâmbios frequentes & 02: intercâmbio razoável \\
\hline & & & 03: intercâmbio frequente \\
\hline & & & 01: uso frequente \\
\hline Uso de insumos externos & Uso de adubos/agrotóxicos & Sem uso & 02: uso eventual \\
\hline & & & 03: sem uso \\
\hline & & & 01: não produz p/ autoconsumo \\
\hline Produção para autoconsumo & Consumo de produtos do lote & $\begin{array}{l}\text { Produz a maior parte dos } \\
\text { alimentos que consome }\end{array}$ & 02: produz a menor parte \\
\hline & & & 03: produz a maior parte \\
\hline 0 & Aplicação de conhecimentos & Jce froo & $\begin{array}{l}\text { 01: sem uso de conhecimentos } \\
\text { locais }\end{array}$ \\
\hline Conhecimentos locais & locais & Uso irequente & 02: pouco uso \\
\hline & & & 03: uso frequente \\
\hline
\end{tabular}




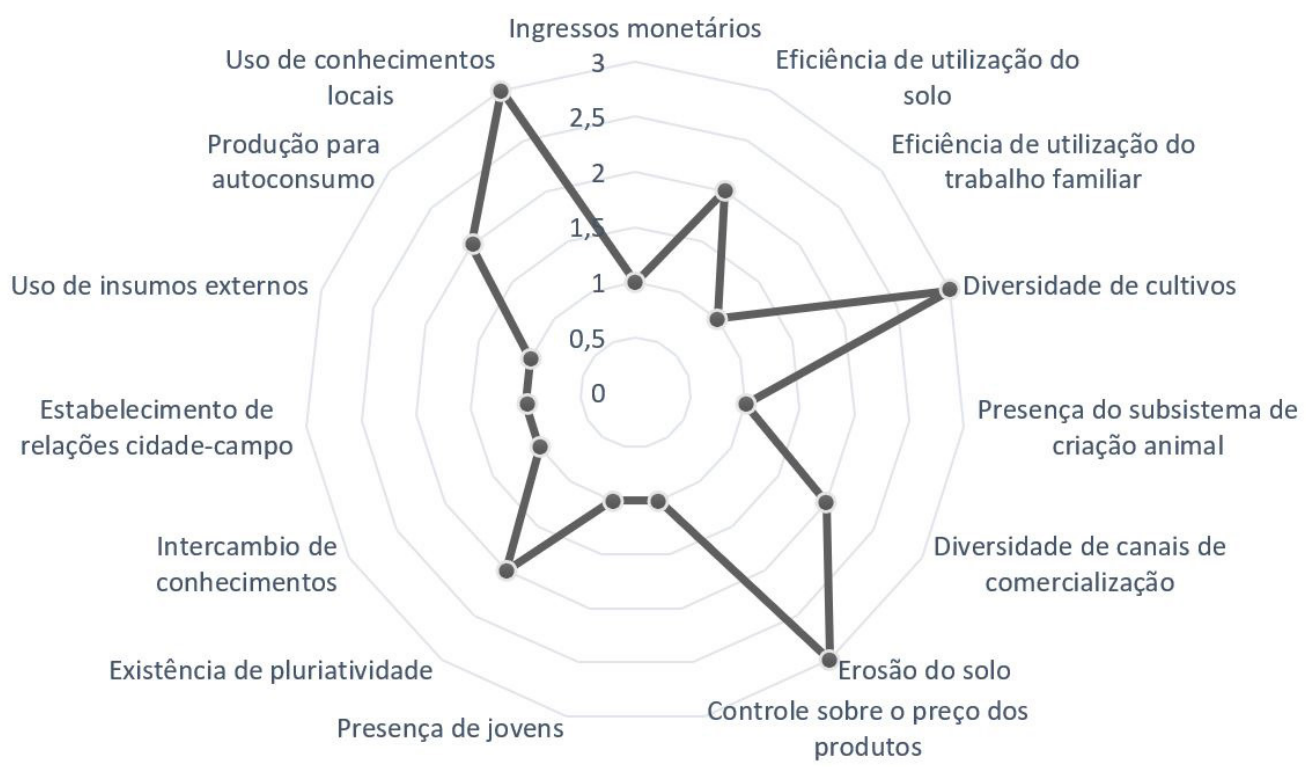

Figura 1. Análise da sustentabilidade no agroecossistema 1 em Baturité/CE, 2019.

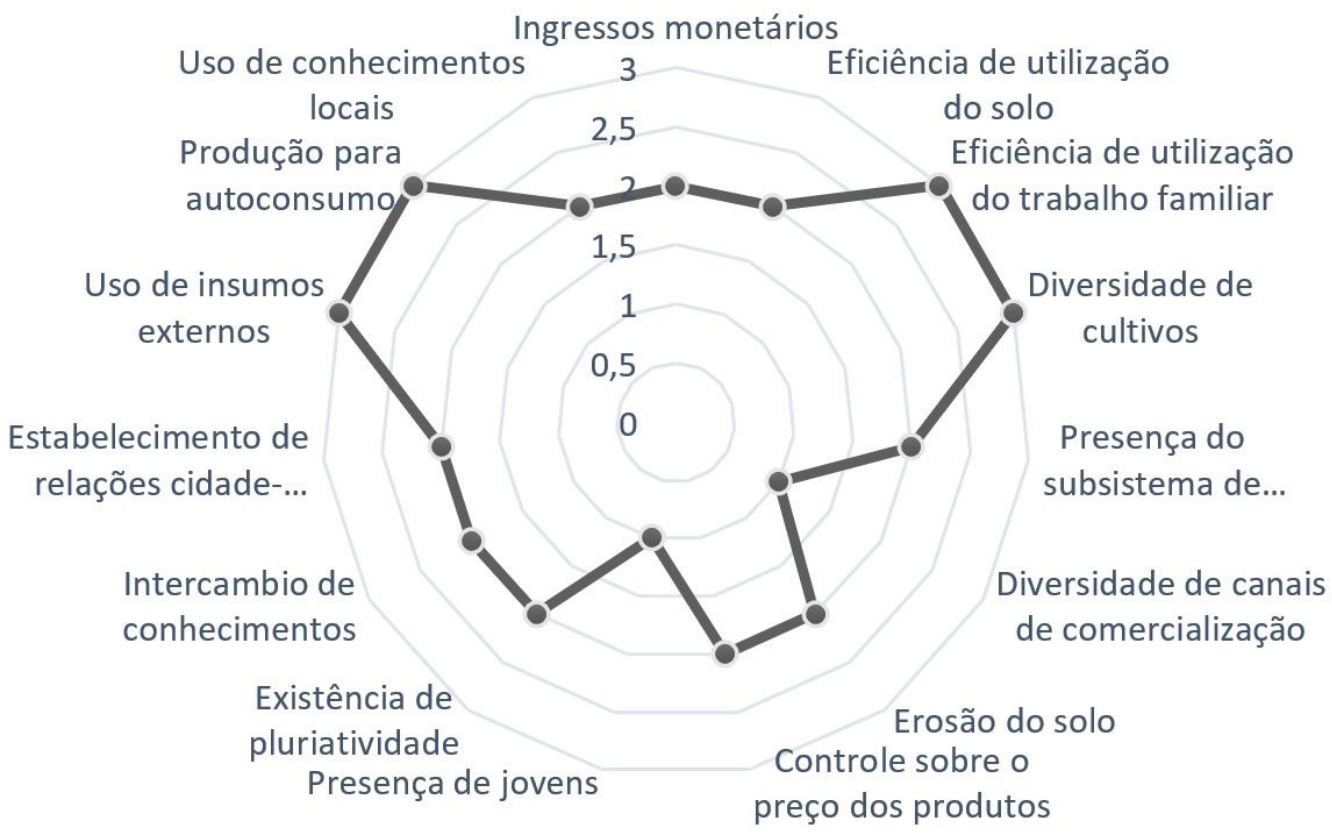

Figura 2. Análise da sustentabilidade no agroecossistema 2 em Barreira/CE, 2019.

\section{Agroecossistema 2 - Barreira/CE}

Este agroecossistema (Figura 2) é composto por uma família (seis pessoas) que detém a propriedade a mais de vinte anos. São provenientes de Barreira, onde trabalham como autônomos em uma fábrica de produção de castanha e uma pessoa possui experiência prévia com agricultura. A propriedade de 8 hectares é ocupada com diversos cultivos: laranja, limão, goiaba, jaca, manga. O componente animal no agroecossistema é uma produção leiteira com fins lucrativos e a produção agrícola não possui canais de comercialização, somente para autoconsumo. A análise da sustentabilidade foi a seguinte:

O uso de técnicas repassadas mediante o tempo de experiência na agricultura é um dos pontos fortes no sistema. A produção agrícola sem fins lucrativos auxilia na pouca entrada de insumos externos, apesar da grande diversificação de cultivos. A produção agrícola e animal contém mão de obra familiar em sua totalidade. A fábrica de beneficiamento de castanha é fonte principal de renda. Os agricultores detêm conhecimento prévio acerca da tabulação preços, porém ainda com sistema arcaico, que prejudica a dinamização financeira.

Como pontos deficitários detemos os canais que são utilizados pelos agricultores familiares para viabilizar a chegada dos alimentos convencionais ou orgânicos na mesa dos consumidores finais Rodrigues et al. (2019). Esses canais são capazes de absorver grande parte da produção obtida, uma vez atendidos os requisitos exigidos para poder fornecer aos 
programas governamentais, centros de distribuição agrícolas ou comércios locais Hickey et al. (2016).

De forma similar a questão do êxodo rural entre os jovens implica num conjunto maior de questões condicionantes Peripolli (2019). Não há como eleger um ou outro aspecto apenas, dada a complexidade das situações. Citam-se fatores educacionais, políticos e tecnológicos. Este indicador deteve notas baixas nos dois agroecossistemas.

A Figura 3 apresenta os índices de sustentabilidade para cada dimensão - econômica (ISEcon), ambiental (ISAmb) e sociocultural (ISSocio) - e para cada agroecossistema. Estes índices foram obtidos somando as notas de cada indicador do conjunto de indicadores econômicos, ambientais ou socioculturais, e depois dividindo esta soma por cinco, já que cada dimensão foi descrita a partir de cinco indicadores.

A partir do gráfico é possível observar que o agroecossistema 2 obteve um melhor desempenho em todas as dimensões. Isso se deve a alguns fatores como: pouco uso de insumos externos, práticas de reaproveitamento de resíduos sólidos, eficiência no trabalho familiar, intercâmbio de conhecimentos, além da fábrica de beneficiamento de castanha advindos de políticas públicas.

Na Figura 4 apresentamos a média de cada uma das dimensões de avaliação, de forma ponderada para todos os agroecossistemas:

A sustentabilidade econômica tem maior déficit nos sistemas estudados nas cidades de Baturité/CE e Barreira/CE, enquanto a sustentabilidade ambiental se sobressai em melhores números.

\section{$\rightarrow$ AGROECOSSISTEMA $2 \multimap$ AGROECOSSISTEMA 1}

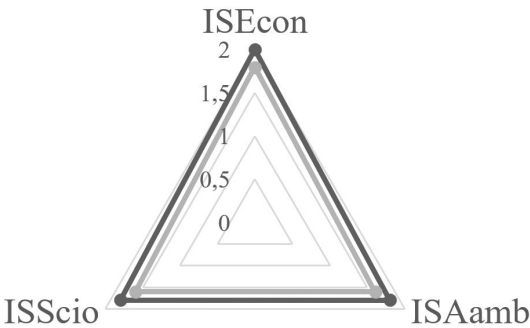

Figura 3. Índices de sustentabilidade para cada dimensão. Maciço de Baturité/ CE, 2019.

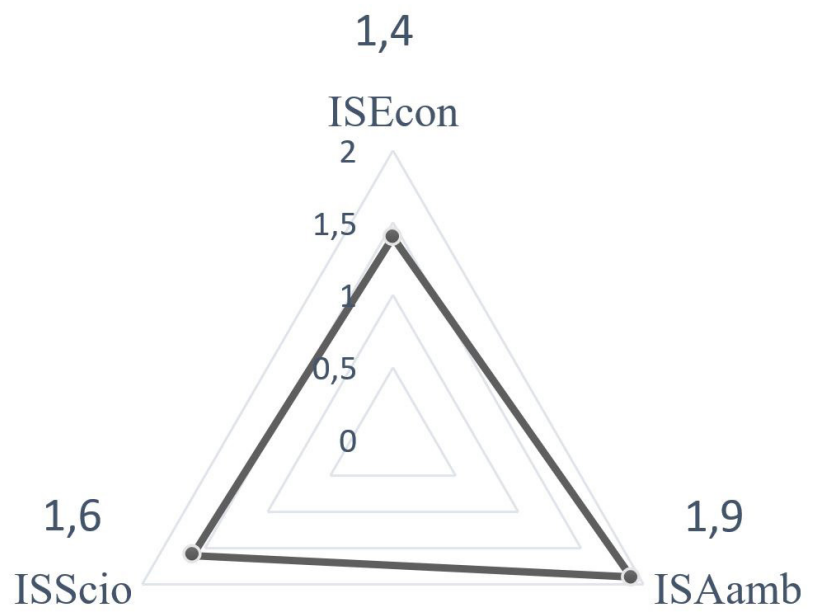

Figura 4. Média das dimensões de avaliação para todos os agroecossitemas. Maciço de Baturité/CE, 2019.
Não há diferenças entre as três dimensões, havendo um equilíbrio entre elas.

\section{Conclusões}

A variabilidade de cultivos do produtor em questão torna esse indicador um é elemento chave para obtenção de maiores índices de sustentabilidade. Neste sentido, os agroecossistemas com presença de culturas diversificadas apresentaram os maiores níveis de sustentabilidade. A monocultura pode implicar em uso intensos de insumos externos como fertilizantes solúveis e agrotóxicos ou como no caso do agroecossistema 1, o hábito da utilização sem deter outra alternativa os problemas propostos.

A partir deste estudo foi possível comparar dois agroecossistemas familiares localizados em uma mesma realidade temporal e espacial (Maciço de Baturité/CE) evidenciando potenciais fraquezas e as integridades dos sistemas. Logo a sustentabilidade econômica dos sistemas deve ser subsidiada com a expansão canais de comercialização de circuitos locais e valorização dos produtos agrícolas associado aos sistemas de cultivos de cada propriedade.

A diversificação de cultivos aliado ao uso mais conservacionista dos recursos disponíveis, com a expansão mercadológica de ambos os sistemas podem auxiliar para a mudança dos níveis de sustentabilidade dos agroecossistemas.

\section{Referências}

Alves, S. F. et al. Indicadores de sustentabilidade para institutos de pesquisa e inovação da área nuclear. Brazilian Journal of Radiation Sciences, Recife, v. 4, n. 1, 2016.

Borges, I. M. S. et al. Family farming: analysis of sustainability through social economic and environmental indicators. Research, Society and Development, Vargem Grande Paulista, v. 9, n. 4, p. e54942832, 2020.

Gallo, A. et al. Indicadores da sustentabilidade de uma propriedade rural de base familiar no estado de Mato Grosso do Sul. Revista Verde de Agroecologia e Desenvolvimento Sustentável, Pombal, v. 11, n. 3, p. 104-114, 2016.

Gallo, A. S. et al. Avaliação da sustentabilidade de uma unidade de produção familiar pelo método MESMIS. Cadernos de Agroecologia, Porto Alegre, v. 9, n. 4, p. 1-12, 2014.

Gonçalves, W. L.; Lira, W. S.; Sousa, C. M. Análise da sustentabilidade da agricultura familiar na produção de tangerina no município de Matinhas, Paraíba. In: CÂNDIDO, G. A.; LIRA, W. S. (Org.). Indicadores de Sustentabilidade para Agroecossistemas: aplicações em diversos tipos de cultivo e práticas agrícolas no estado da Paraíba. Campina Grande: EDUEPB, 2016. p. 99-160.

Guimarães, N. F. et al. Avaliação da sustentabilidade de um agroecossistema pelo método MESMIS. Scientia Plena, Aracaju, v.11, n.5, p.1-11, 2015.

Hickey, G. M. et al. Quantifying the economic contribution of wild food harvests to rural livelihoods: a global-comparative analysis. Food Policy, USA, v. 62, p. 122-132, 2016.

INSTITUTO BRASILEIRO DE GEOGRAFIA E ESTATÍSTICA IBGE. Sistema IBGE de Recuperação Automática-SIDRA. Rio 
de Janeiro: IBGE, 2020. Disponível em: <http://www.sidra.ibge. br>. Acesso em: 22 maio de 2020.

INSTITUTO DE PESQUISA E ESTRATÉGIA ECONÔMICA DO CEARÁ - IPECE. Secretaria do Planejamento e Coordenação (SEPLAN). Perfil das macrorregiões do Ceará. Fortaleza: IPECE, 2006. Vol. 14.

Masera, O.; Astier, M.; López-Ridaura, S. Sustentabilidad y manejo de recursos naturales: el marco de evaluación MESMIS. México: GIRA/Mundi-Prensa, 2000. 109 p.

Medeiros, A. F. Q. et al. Controle e apuração de resultado na agricultura familiar sob a ótica da sustentabilidade de produtores rurais. Custos e Agronegócio, Recife, v. 8, n. 3, 2012.

Panno, F.; Dessimon Machado, J. Influências na Decisão do Jovem Trabalhador Rural: Partir ou Ficar no Campo. Desenvolvimento em Questão, Ijuí, v. 12, n. 27, p. 264-297, 2014.
Peano, C. et al. Evaluating the sustainability in complex agri-food systems: The SAEMETH framework. Sustainability, Switzerland, v. 7, n. 6, p. 6721-6741, 2015.

PERIPOLLI, O. J. O processo de esvaziamento do campo entre Jovens Camponeses: os desafios colocados à escola. Revista da Faculdade de Educação (Universidade de São Paulo), v. 14, n. 16, p. 77-93, 2019.

Rodrigues, B. A.; Bittencourt, J. V. M.; Macedo, L. M. Canais de comercialização para os alimentos orgânicos: um estudo no Sul do Brasil. Cadernos de Ciência \& Tecnologia, Brasília, v. 36, n. 2, p. 26441, 2019.

SILVA, G. M. et al. Indicadores de sustentabilidade na visão de agricultores familiares como instrumento para gestão de unidades de produção com pecuária de leite. In: CONGRESSO DA SOCIEDADE BRASILEIRA DE SISTEMA DE PRODUÇÃO, 11., 2016, Pelotas. Anais... Pelotas: SBSP, 2016. 